\title{
The COVID-19 Pandemic: Defining the Clinical Syndrome and Describing an Empirical Response
}

\author{
Mandalam S. Seshadria and T. Jacob John ${ }^{b}$ \\ ${ }^{a} \mathrm{MD}, \mathrm{PhD}, \mathrm{FRCP}$, Former Professor of Medicine \& Endocrinology, Department of Endocrinology Diabetes \& \\ Metabolism, Christian Medical College \& Hospital, Vellore; Consultant Physician \& Endocrinologist, Honorary \\ Medical Director, Thirumalai Mission Hospital, Vanapadi Road, Ranipet, India \\ b PhD (Virology), FRCP (Paediatrics), Former Professor\& Head, Department of Clinical Virology, Christian Medical \\ College \& Hospital, Vellore, India
}

\begin{abstract}
The novel corona virus infectious disease, COVID-19, is a pandemic now and is raging through several continents, posing a challenge to health-care systems of all the countries and disrupting lives and livelihoods across the world. The facilities for virus testing are available for only limited numbers in each country and each country excludes a large number of potentially infected subjects because the lab test is done for only certain categories. Nearly $80 \%$ of those infected will, therefore, go undiagnosed. There is an urgent need, therefore, to define the clinical syndrome so that practitioners at the primary and secondary levels can make a confident, clinical diagnosis and proceed to manage patients early and effectively. Chloroquine and hydroxychloroquine, both antimalarials, have shown promise in limited trials in France and China. They are inexpensive, have been around for several decades in the prevention and treatment of malaria, have well-known side-effects and, in the shortterm, safe for use. We propose that practitioners make a preliminary clinical diagnosis of the COVID-19 syndrome based on simple clinical criteria and lab tests and proceed to manage patients and protect other family members and contacts by using isolation measures and short regimens of these anti-malarial and other medications, anticipating results of more clinical trials.
\end{abstract}

Key Words: COVID-19, clinical syndrome, empirical therapy, hydroxychloroquine.

\section{Introduction}

The COVID-19 pandemic sweeping across the world is continuing to take a heavy toll in terms of human lives and is threatening the global economy. It is currently spreading rapidly across several continents, and the peak is yet to come. The variable sensitivity of the polymerase chain reaction (PCR) based tests employed, different criteria for testing in different countries, limited availability of the testing facilities, and the high 
cost of testing will result in underdiagnosis. ${ }^{1}$ Therefore, alternative approaches that do not rely on testing everyone with fever and cough with PCR are necessary in low- and middle-income countries.

\section{Diagnosis}

In clinical medicine, a syndrome may be diagnosed using specific clinical and commonly available laboratory criteria, especially when the situation is a medical emergency and treatment is urgent. To cite a common example, empirical use of antibiotics, pending culture reports, is standard practice in treating sepsis syndrome, and completion of a course of antibiotics is indicated if there is a clinical response, even if the cultures eventually turn out to be negative. ${ }^{2}$

In the face of a pandemic, the possibility of contact with infected patients (typical epidemiologic setting) is a very important element for defining the clinical syndrome. When a disease has a high, attack rate, a clinical diagnosis may provide a more sensitive approach than a lab test. Further, in the context of resource poor countries, only select patients are tested by PCR. Therefore, relying only on laboratory tests for a diagnosis of COVID-19 will grossly underestimate the true disease prevalence and incidence.

In view of this, it is reasonable to assume that every individual that meets the clinical case definition inclusion criteria is presumptively positive for COVID-19 and to treat as such. This will provide a more sensitive approach and ensure that most of those who actually have the disease are detected and treated. Such an approach will be in the best interests of both individual patients and the community as a whole. In the public health systems of countries like India, where access to lab tests is more difficult than access to an outpatient consultation, this approach would be easier to implement.

April 2020. Christian Journal for Global Health, 7(1)
While the WHO case definition ${ }^{3}$ is simple to use, it does not give adequate importance to fever which is the most common symptom ${ }^{4}$ and ignores smell and taste disturbances that appear to be unique to this viral illness. ${ }^{5}$ In the COVID-19 clinical syndrome, fever is observed in $>95 \%$ of individuals and selecting this symptom as a criterion will ensure that the clinical definition will have good sensitivity. Sudden, otherwise unexplained loss of smell and taste ${ }^{5}$ occurs in about $34 \%$ of COVID-19 patients and including this unique symptom will confer greater specificity for the clinical diagnosis. In the light of these arguments, we recommend that the clinical syndrome be defined using criteria as listed below:

\section{Mandatory criterion:}

Fever of three or more days duration without other obvious localizing symptoms such as dysuria, skin, or soft tissue infections

\section{Major criteria:}

1. Dry cough

2. Sudden recent onset loss of smell and or taste sensation

(anosmia due to nasal block and sinusitis to be excluded)

3. Physical findings of crepitations on chest auscultation

4. Chest X Ray showing peripheral patchy infiltrate (not lobar pneumonia or cavitating lesion)

5. Respiratory rate $>25 /$ minute

\section{Minor criteria:}

1. Diarrhoea

2. Severe headache, body aches (myalgia)

3. Fatigue and lassitude

4. Normal or low normal total WBC count and lymphopenia (Lymphocytes $<20 \%$ on differential count) 


\section{Epidemiologic setting:}

(When there is community spread this criterion may not be useful):

1. Travel within the past four weeks to or from any other country or a big crowded city in the country.

2. Visit within the last four weeks to a crowded place such a bus stand, railway station, movie theatre, airport, place of worship, etc., without a mask and or without maintaining a physical distance of two meters

3. Contact with a case of COVID-19 at home or at work-place.

The clinical syndrome can be presumed if, in the presence of the mandatory criterion (fever), the following criteria are met:

1. Presence of one epidemiologic setting along with two major criteria or one major criterion and two minor criteria

2. Even in the absence of the epidemiologic setting, the presence of three major criteria and two minor criteria or two major criteria and three minor criteria

Where available, a positive PCR lab test, in combination with the clinical syndrome criteria, offers confirmation of diagnosis while a negative PCR test does not necessarily negate the diagnosis due to less than optimal test sensitivity. In fact, PCR and CT thorax combined have higher sensitivity than either test alone for diagnosing serious COVID-19 infection. ${ }^{6}$

Therefore, in resource poor settings, we can consider two groups of subjects:

a) those having the COVID-19 clinical syndrome (large numbers)

b) cases confirmed by PCR testing (smaller numbers)

For those with the clinical syndrome, if feasible, nasopharyngeal swabs, or even throat swabs can be sent to a regional laboratory for

April 2020. Christian Journal for Global Health, 7(1) confirmation. In endemic malarial zones, malaria should be excluded by a rapid test and peripheral smear.

\section{Isolation and Prevention of Spread}

Pending results, clinical management should be initiated as set out below:

Isolate affected subjects at home for a period of 21 days (three weeks). Get a younger member of the family aged less than 45 to be the primary care-giver. A detailed isolation procedure at home, as spelt out below, has to be strictly followed to prevent within family spread. Other family members, in particular, the elderly, those with diabetes, and cardiac disease should also home quarantine for four weeks (to allow for incubation period and duration of viral shedding) to prevent serious disease in them. Younger family members can go out to get essential requirements but wear a mask when they do so and maintain a physical distance of two meters from others to prevent community spread. (See Appendix A for our example of home isolation procedure).

Any member of any family that develops a fever, cough, and cold should not panic and go to hospital unless there is significant breathing difficulty. Other respiratory viruses such as influenza $(5-20 \% \text { of the population each year })^{7}$ and the common cold are highly prevalent and must be considered in the differential diagnosis. The hospital may be crowded with other sick patients who may have COVID-19. It is essential that every household has simple medications such as paracetamol for fever and an antihistamine such as pheniramine or cetirizine which may minimize sneezing and limit nasal discharge. These supplies are better issued to individual households by the local civil administration and or by local nongovernmental agencies with instructions for use, so that crowding at hospitals and medical shops is avoided. The mobile phone number of an 
individual in the family can be made available to the proximate primary or secondary level hospital so that the lab test reports, when they arrive, can be communicated. A designated mobile number at the health care facility can be provided to the family. The family can contact this number in the event of any worsening or questions. The follow up information can be recorded on spread-sheet.

With the crowded living conditions in most middle- and low-income households, these quarantine measures will prove to be major challenges; isolation may not be possible in poor households living in one or two rooms. The local administration needs to face this reality and design isolation facilities near home, such as a school building, if possible. The Government and nongovernmental organisations (NGOs) should ensure essential supplies to these quarantined families so that they can effectively practice what is recommended.

\section{Treatment}

The antimalarial drugs, chloroquine and hydroxychloroquine (HCQ), have shown some efficacy in in-vitro experiments. Limited observational studies using HCQ in infected subjects in France and China have been shown to reduce virus load and also hasten virus clearance from two weeks to six days. ${ }^{8,9}$ The proposed mechanisms of action of these drugs ${ }^{9}$, such as interference with adhesion of virus to cell surface receptor, inhibition of viral replication by increasing the $\mathrm{pH}$ in the endo-lysosomes, and an anti-inflammatory action (to reduce cytokine production and immunologically mediated inflammation), imply that the drugs may be of use early in the course of infection as well as in the delayed cytokine storm. ${ }^{10}$

However, in the absence of controlled clinical trials in severe cases of COVID-19 pneumonia, some suggest that these drugs should only be used in randomized controlled clinical trials. ${ }^{11}$ Major

April 2020. Christian Journal for Global Health, 7(1) clinical trials have just started, and the results will probably be available only after about 12 weeks by which time the pandemic may be waning. ${ }^{12}$

Physicians working in endemic malarial zones have good experience with these drugs. However, in non-endemic zones, HCQ is commonly used for rheumatological disorders and for malaria prophylaxis in travellers. In New York and other hot spots for COVID-19, physicians have started using these drugs on an empirical basis for treating severe COVID-19 pneumonia. In countries such as India, adequate supplies of chloroquine and HCQ are available, and the Government and NGOs can cope with the demands for these drugs during this pandemic without compromising supplies of the drug for patients with rheumatological disorders who need the drug.

In rural settings, for reasons mentioned earlier, physicians may need to resort to syndromic diagnosis and institute empirical management protocols for sick patients, and if there is good clinical improvement, complete the course of HCQ. Perhaps based on the safety profile of once weekly doses of the drug in malaria prophylaxis, the relatively short duration of time for which chemoprophylaxis may be required, the suggested dosage schedules, which are similar to doses used for malaria (treatment and prophylaxis), and the potential for reduction of infectivity, the Indian Council of Medical Research (ICMR) has recommended prophylaxis with HCQ for frontline health care workers and household contacts of SARS-CoV-2 positive subjects. ${ }^{13}$

When a physician chooses to use HCQ for either empirical treatment for COVID-19 or chemo-prophylaxis as per Government guidelines, due precautions are mandatory for the elderly, those with diabetes and cardiac disease, in whom dosing has to be modified to avert potential side effects of the drug. An outline of suggested empirical treatment, chemoprophylaxis, monitoring, and precautions is detailed below: 


\section{Empirical HCQ Treatment}

(Effective dose for treatment derived from pharmacokinetics-based computer assisted modelling $)^{14}$

Hydroxychloroquine $200 \mathrm{mg}, 2$ tablets, Q12h (total $800 \mathrm{mg}$ ) on day 1 followed by $200 \mathrm{mg}, 1$ tablet, Q12h (total $400 \mathrm{mg}$ per day) for 4 more days.

a. Youngsters without any risk factors: Monitor progress of clinical illness daily (over mobile phone). Maintain a database on a spread-sheet, and avoid HCQ in mild to moderately severe disease.

b. Avoid HCQ in those with chronic renal or liver disease.

c. In subjects with diabetes mellitus:

- While on HCQ, treatment, reduce dose of anti-diabetic drugs by $25-30 \%$ in order to avert hypoglycaemia.

- Institute home monitoring or field monitoring of blood sugars by glucometer during HCQ treatment. Further dose adjustment of anti-diabetic drugs can be based on plasma glucose values

- Once the treatment course is finished, over the next 3-7 days get back to the previous stable dose of oral anti-diabetic drugs and or insulin

d. Those with cardiac disease on medication:

Look at the drug list, check for potential drug interactions, and make a considered decision in consultation with the attending cardiologist. Baseline ECG (focus on corrected QT interval) and ECG on alternate days until course is over would be useful; however, this may necessitate hospital admission. Monitor serum electrolytes and magnesium and correct hypokalaemia and hypomagnesemia when detected.

April 2020. Christian Journal for Global Health, 7(1)

\section{Zinc Supplementation}

In vitro studies have shown that intracellular zinc, when present in sufficient concentration, inhibits viral replication and chloroquine acts as an ionophore, facilitating transport of zinc from extracellular to intracellular compartment. Therefore, elemental zinc of $50 \mathrm{mg}$ per day, orally, once daily can be co-prescribed with HCQ. ${ }^{15,16}$

\section{Use of Antibiotics}

In order to treat secondary bacterial infection which occurs in about $50 \%$ of COVID- 19 cases, Azithromycin $500 \mathrm{mg}$, once daily, for 5 days or Amoxicillin/potassium-clavulanate $625 / 125 \mathrm{mg}$ Q12 hourly, for 5 days (common antibiotics used for treating community acquired pneumonia) may be added at the discretion of the treating physician based on a persistent fever $>38$ degree Celsius and productive cough persisting beyond 5 days.

\section{Convalescent plasma for severely ill COVID- 19 patients}

Encouraging observational reports in small numbers of patients have aroused wide-spread interest in the use of convalescent plasma for severely ill COVID-19 patients on ventilators. ${ }^{17}$. Randomized controlled clinical trials have commenced with this treatment approach. In general, in viral illnesses antibody response is much brisker in those who have a clinical illness than in those with asymptomatic or subclinical illness. ${ }^{18}$ Patients with clinically diagnosed COVID-19 syndrome may be the ones with the highest titres of antibodies. Utilising the syndromic approach may help identify potential plasma donors in resource poor settings. Since those with the clinical syndrome will be the larger number than those with PCR-proven SARS-CoV2 infection, a physician can select willing individuals who have recovered from the clinical syndrome for checking on antibody titres prior to plasmapheresis and, thus, reduce costs. 


\section{Preventive Treatment}

House-hold contacts of the subject with clinical diagnosis of COVID 19 syndrome (During the lock down period in India, household members will predominate), those working or interacting closely with the index case in the workplace (such as grocery store, post office, bank, etc. ) and those in migrant groups amongst whom one individual has been presumptively diagnosed to have COVID-19 clinical syndrome or confirmed to have COVID-19 by PCR will be the contacts.

Regimen for household and other contacts (as per ICMR advisory ${ }^{13}$ ):

HCQ $200 \mathrm{mg}, 2$ tabs, twice daily (800 mg per day) for day 1 followed by $200 \mathrm{mg}, 2$ tabs, once a week (400 mg per week) for the next several weeks

Preventive treatment for contacts starts as soon as the clinical case is diagnosed. If not, it can be started any time up to day 14 of presumptive diagnosis in the index patient. Long-term side effects, like retinopathy, are dose and duration dependent and are unlikely during these short-term treatment protocols.

\section{Conclusion}

Protocols by the Kerala, Tamil Nadu, Maharashtra State Governments, and the guidelines from the All India Institute of Medical Sciences, New Delhi, adequately cover management of confirmed cases. We highlight the need to address the clinical COVID-19 syndrome, where PCR testing may not be performed because of the restrictive selection criteria for PCR testing or lack of availability. For such a syndromic approach to be effective would require a quick nation-wide implementation during the shelter or lock-down period.

Governments and NGOs in other middle- and low-income countries involved in responding to the health care challenge posed by COVID-19 should consider implementing the syndromic

April 2020. Christian Journal for Global Health, 7(1) approach. They should mobilize material and human resources and medication to do this quickly through their networks of healthcare professionals.

We note the use of HCQ has not yet been established in clinical trials and in the results of the multinational and multi-centric clinical trial "Solidarity" 12 will probably not be available until after the pandemic. We should be closely monitoring the latest evidence for HCQ, but in the absence of data from controlled clinical trials and given the expected burden of mortality from Covid 19 , the treatment of the clinical syndrome and use of chemoprophylaxis for contacts of the presumed COVID-19 syndrome (rather than only confirmed cases and their contacts) seems important in countries and settings with resource constraints.

\section{References}

1. Patel R, Babady E, Theel ES, Storch GA, Pinsky

BA, St. George K, et al. Report from the

American Society for Microbiology COVID-19

International Summit, 23 March 2020: value of

diagnostic testing for SARS-CoV-2/COVID-19.

2020. mBio 11:e00722-20. Available from: https://doi.org/10.1128/mBio.00722-20

2. Hotchkiss RS, Moldawer LL, Opal SM, Reinhart

K, Turnbull IR, Vincent J-L. Sepsis and septic shock. Nat Rev Dis Primers. 2017;2:16045.

https://doi.org/10.1038/nrdp.2016.45

3. World Health Organization. Health topics:

Coronavirus [Internet]. Available from:

https://www.who.int/healthtopics/coronavirus\#tab=tab 3

4. Wang D, Hu B, Hu C, Zhu F, Liu X, Zhang J,Wet al. Clinical characteristics of 138 hospitalized patients with 2019 novel coronavirus-infected pneumonia in Wuhan, China. JAMA. 2020. 323:1061-69.

https://doi.org/10.1001/jama.2020.1585

5. Giacomelli A, Pezzati L, Conti F, Bernacchia D, Siano M, Oreni L, et al. Self-reported olfactory and taste disorders in SARS-CoV-2 patients: a cross-sectional study. Clinical Infectious Diseases. 2020 Mar 26; ciaa330.

https://doi.org/10.1093/cid/ciaa330 
6. Ren X, Liu Yan, Chen, H, Liu W, Guo Z, zhang $\mathrm{y}$, et al. Application and optimization of RT-PCR in diagnosis of SARS-CoV-2 infection. Preprints with The Lancet. 2020 Mar 3.

https://doi.org/10.2139/ssrn.3546086

7. Thomas J. The flu: facts, statistics, and you [Internet]. Healthline. 2018 Nov 19. Available from:

https://www.healthline.com/health/influenza/facts -and-statistics\#1

8. Gautret P, Lagier JC, Parola P, Hoang VT, Meddeb L, Mailhe M, Doudier B, et al. Hydroxychloroquine and azithromycin as a treatment of COVID-19: results of an open-label non-randomized clinical trial. Int J Antimicrob Agents, 2020 Mar 20:105949. [Epub ahead of print]. https://doi.org/10.1016/j.ijantimicag.2020.105949

9. Singh AK, Singh A, Shaikh A, Singh R, Misra A. Chloroquine and hydroxychloroquine in the treatment of COVID-19 with or without diabetes: a systematic search and a narrative review with a special reference to India and other developing countries. Diabetes \& Metabolic Syndrome, Clinical Research \& Reviews PII. S18714021(20)30051-5. https://doi.org/10.1016/j.dsx.2020.03.011

10. Savarino A, Boelaert JR, Cassone A, Majori G, Cauda R. Effects of chloroquine on viral infections: an old drug against today's diseases. Lancet Infect Diseases. 2003.3:722-7. https://doi.org/10.1016/S1473-3099(03)00806-5

11. Yazdany J,Kim AHJ. Use of hydroxychloroquine and chloroquine during the Covid-19 pandemic: what every clinician should know. Annals Intern Med. 2020. https://doi.org/10.7326/M20-1334

12. WHO. Global research on coronavirus disease (COVID-19)/ "Solidarity" clinical trial for
COVID-19 treatments [Internet]. Available from: https://www.who.int/emergencies/diseases/novelcoronavirus-2019/global-research-on-novelcoronavirus-2019-ncov/solidarity-clinical-trialfor-covid-19-treatments

13. Indian Council of Medical Research, National Task Force for COVID-19. Advisory on the use of hydroxyl-chloroquine as prophylaxis for SARSCoV-2 infection [Internet]. Available from: https://www.mohfw.gov.in/pdf/Advisoryontheuse ofHydroxychloroquinasprophylaxisforSARSCoV 2infection.pdf

14. Yao X, Ye F, Zhang M, Cui C, Huang B, Niu P, et al. In vitro antiviral activity and projection of optimized dosing design of hydroxychloroquine for the treatment of severe acute respiratory syndrome coronavirus 2 (SARS-CoV-2). Clinical Infectious Diseases. 2020 Mar 9; ciaa237. https://doi.org/10.1093/cid/ciaa237

15. Aartjan JW, te Velthuis AJW, van den Worm SHE, Sims AC, Baric RS, Snijder EJ, et al. Zn2+ inhibits coronavirus and arterivirus RNA polymerase activity in vitro and zinc ionophores block the replication of these viruses in cell culture. PLoS Pathogens. 2020 Nov. https://doi.org/10.1371/journal.ppat.1001176

16. Jing Xue J, Moyer A, Peng B, Wu J, Hannafon $\mathrm{BN}$, Ding W-Q. Chloroquine is a zinc ionophore. PLoS ONE 9(10): e109180.

https://doi.org/10.1371/journal.pone.0109180

17. Duan K, Liu B, Li C, Zhang H, Yu T, Qu J,et al. Effectiveness of convalescent plasma therapy in severe COVID-19 patients. PNAS. 2020 Apr 6. https://doi.org/10.1073/pnas.2004168117

18. Ganem D, Prince AM. Hepatitis B virus infection: natural history and clinical consequences. $\mathrm{N}$ Engl J Med. 2004;350:1118-29. https://doi.org/10.1056/NEJMra031087

Peer Reviewed: Submitted 3 Apr 2020; Accepted 21 Apr 2020; Published 27 Apr 2020

Competing Interests: None declared.

Acknowledgements: Thirumalai Mission Hospital, Ranipet, India.

Correspondence: Dr. Seshadri, India. mandalam.seshadri@gmail.com

April 2020. Christian Journal for Global Health, 7(1)

CHRISTIAN JOURNAL GLOBAL HEALTH 
Cite this article as: Seshadri MS, John TJ. The COVID-19 pandemic: Defining the clinical syndrome and describing an empirical response. Christian Journal for Global Health. April 2020;7(1):37-44. https://doi.org/10.15566/cjgh.v7i1.365

(C) Authors This is an open-access article distributed under the terms of the Creative Commons Attribution License, which permits unrestricted use, distribution, and reproduction in any medium, provided the original author and source are properly cited. To view a copy of the license, visit http://creativecommons.org/licenses/by/4.0/

\section{Appendix A: Home Isolation Procedure (from Thirumalai Mission Hospital)}

The clinically diagnosed COVID-19 patient should wear a mask, wear full sleeves, and cough or sneeze only into a disposable tissue or into the crook of the elbow and not into the hand. He should be in a wellventilated single room preferably with an attached bathroom, not shared with others. Where there is a need to share a bathroom, the surfaces that the patient has touched should be cleaned with dilute bleach or soap and water and mopped dry after use by the affected person. The patient's toilet kit (tooth-brush, shaving kit, etc.) should be kept separate and other house-hold members avoid coming in contact with these items of personal use. The patient's clothes should be washed thoroughly with soap and water daily, dried, and kept separate for him/her to re-use. Bed linen should be similarly washed and dried at least once in 3 days and kept separate. If the patient is using a mobile phone, it should not be shared with others and the surface wiped clean carefully with tissue moistened with hand sanitizer 3-4 times per day. Other family members also use a mask all the time at home and maintain a physical distance of two meters from the patient, avoiding physical contact such as shaking hands, patting on the back, hugging, etc. The household members should avoid visiting others and not allow visitors until the quarantine period is over. Communication with others outside the family should be by phone or messages (SMS). If there are people above age 65 in the household, ensure that they follow all the precautions that have been advised for the affected patient. Only younger unaffected members of the family (Age $<45)$ should go out for buying provisions, wearing a mask when

April 2020. Christian Journal for Global Health, 7(1) going out to shops, maintaining the critical physical distance of two meters from other people on the road and in the shop, and avoiding standing in groups to talk. Children in the house can play indoor games with other family members, read, paint, and listen to or play music for pastime. Children should also wear masks and maintain a physical distance of 2 meters from the affected individual. Every member of the household should practice frequent and thorough hand washing with soap and water after they come in contact with door knobs, lift buttons, and other potentially contaminated surfaces. If there is a care-taker for the elderly, it is the responsibility of the residents of the house to instruct the care-taker to wear a mask all the time, to use a pair of gloves while working, to sanitize gloves at the end of the work, to practice thorough hand washing with soap and water after they have finished their work and before they help elders, to avoid unnecessarily hanging around in common areas, and to abstain from work for 3 weeks if the care taker or his or her family member has a febrile illness. It is important that care-takers are paid their wages when they or their family members fall sick. 